\title{
Stability of Positively Charged Nanoemulsion Formulation Containing Steroidal Drug for Effective Transdermal Application
}

\author{
Stephanie Da Costa, ${ }^{1}$ Mahiran Basri, ${ }^{1,2}$ Norashikin Shamsudin, ${ }^{3}$ and Hamidon Basri ${ }^{3}$ \\ ${ }^{1}$ Department of Chemistry, Faculty of Science, Universiti Putra Malaysia, Selangor, Malaysia \\ ${ }^{2}$ Laboratory of Molecular Biomedicine, Institute of Bioscience, Universiti Putra Malaysia, Selangor, Malaysia \\ ${ }^{3}$ Department of Medicine, Faculty of Medicine and Health Sciences, Universiti Putra Malaysia, Selangor, Malaysia \\ Correspondence should be addressed to Mahiran Basri; mahiran@upm.edu.my
}

Received 22 July 2013; Revised 15 April 2014; Accepted 23 April 2014; Published 15 July 2014

Academic Editor: Isabel Seiquer

Copyright (C) 2014 Stephanie Da Costa et al. This is an open access article distributed under the Creative Commons Attribution License, which permits unrestricted use, distribution, and reproduction in any medium, provided the original work is properly cited.

\begin{abstract}
This paper emphasizes the formation of a positively charged nanoemulsion system for steroid drugs (hydrocortisone). It is believed that positively charged nanoemulsion provides more effective penetration of the skin. Therefore in our study we focused on the incorporation of phytosphingosine which serves as a positively charged cosurfactant in the nanoemulsion system. Negatively charged nanoemulsions were formulated mainly for comparison. Freshly prepared formulations were formed with particle size less than $300 \mathrm{~nm}$ and showed good stability over time. The oil-in-water nanoemulsion also showed good viscosity, conductivity, and $\mathrm{pH}$ values. From TEM micrograph, particle size showed consistent results with the measurement using photon correlation spectroscopy. It was concluded that both positively and negatively charged nanoemulsions showed good stability and have great potential in transdermal delivery system. Though, further investigation of the drug release and drug penetration of both positively and negatively charged nanoemulsions will be studied to further prove the efficacy of nanoemulsion with hydrocortisone as a delivery system for dermal application.
\end{abstract}

\section{Introduction}

In the recent years, there has been a growing interest in utilizing steroidal anti-inflammatory drugs (SAIDs) to reduce pain and inflammation. Hydrocortisone, less potent SAID, is commonly used to treat atopic dermatitis. Due to its less potent behavior, hydrocortisone only gives minimal side effects. However, long term administration would result in serious side effects such as gastrointestinal ulcers and gastrointestinal bleeding if administered orally [1]. Hence a transdermal application route was chosen in this study.

Transdermal drug delivery system (TTDS) is one of the most effective routes for hydrophobic drugs and presents many advantages. This system is known to enhance bioavailability of drug, maintains the plasma drug level for a longer period [2], and reduces side effects. Besides that, with topical administration, immediate withdrawal of treatment is possible if there are signs of incompatibility. Transdermal route would be ideal because it is more convenient, thus making the administration easier.

For an effective delivery through the topical route, the delivery system must have a high drug permeation rate. This is an obstacle faced by TTDS. Insufficient drug penetration is one of limiting factors in transdermal application of most drugs. Less drug penetration would result in a lower pharmacological effect [3], hence no improvement on the target site. Difficulty in drug penetration through the skin is due to the fact that skin consists of many layers.

Stratum corneum, the outer layer of the skin, plays a role as a skin barrier. The horny layer of the stratum corneum restricts the penetration of drug either through the intercellular route or transcellular route. The latter is a more direct route. In order to have successful penetration, hydrocortisone has to pass through not only lipophilic but also hydrophilic 
substances [4], therefore making drug permeation almost impossible.

In order to overcome this problem, a positively charged cosurfactant, phytosphingosine, is added into the delivery system. Since skin surface is negatively charged because of the existing protein residues, it is more prone to solutes that carry a positive charge [5]. Phytosphingosine is a sphingoid base that is naturally present in the human body [3]. This cosurfactant also takes part in the formation of ceramides which is vital in promoting skin hydration especially in atopic dermatitis [6-8].

Nanoemulsion has always been a promising vehicle for transdermal delivery. With the existing small particle size in the range of 100-600 $\mathrm{nm}$ [9], which gives large surface area of the system and low interfacial tension of the droplets, nanoemulsions are able to penetrate through the skin easily. Besides that, nanoemulsions are thermodynamically stable but kinetically unstable. In other words, nanoemulsion could resist phase separation, hence being more stable and having longer shelf life [10].

The primary aim of this study is to formulate, characterize, and compare a positively charged and a negatively charged transdermal delivery system for hydrocortisone. Therefore in the whole study we focused on the effect of phytosphingosine, the positive cosurfactant used, on particle size, zeta potential, and stability of the nanoemulsion system for a period of $3 \mathrm{~m}$ (months).

\section{Materials and Methods}

2.1. Materials. Hydrocortisone was purchased from Alfa Aesar (Britain). Lipoid S75 was obtained from Sigma-Aldrich (Munich, Germany) and Tween 20 from Merck (Hohenbrunn, Germany). Palm kernel oil esters (PKOEs) were used as the oil phase while deionized water as the aqueous phase. Both PKOEs and deionized water were prepared in our laboratory. Phytosphingosine was purchased from Evonik Industries (Germany). The solvents used were ethanol (EtOH) and isopropanol (IPA) which were obtained from SigmaAldrich chemicals. A preservative, phenonip, was obtained from Gattefosse (North America, USA).

2.2. Solubility Test. The solubility of hydrocortisone in water, palm kernel oil esters, phosphate buffer at $\mathrm{pH} 7.4$, isopropanol, ethanol, ethyl acetate, and series of surfactants (Tween 20, Tween 40, Tween 65, Span 80, and Span 20) was determined by adding excess amount of drug to $20 \mathrm{~mL}$ of these solutions. The mixture was stirred with a magnetic bar for $24 \mathrm{~h}$. The solution was then filtered using polyvinylidene difluoride (PVDF), 0.22 and $0.45 \mu \mathrm{m}$, Milipore filter paper, and analyzed by high performance liquid chromatography (HPLC). The Tween and Span series chosen as nonionic surfactants have been identified as those with the least toxicity and irritant potential [11].

2.3. Construction of Ternary Phase Diagram. Palm kernel oil esters and surfactants (Lipoid S75 and Tween 20) were weighed at various ratios ranging from $0: 100$ to $100: 0$
(PKOEs+Lipoid S75: Tween $20(60: 40))$. More mixture was prepared in the vicinity of the phase boundaries in order to accurately determine their positions. The ternary phase diagram was developed using aqueous titration method. Water $(5 \%(\mathrm{w} / \mathrm{w}))$ was added incrementally to the mixtures. The mixture was placed in a $10 \mathrm{~mL}$ Teflon screw-cap glass tube, sealed, and homogenized with vortex mixer (Model VTX3000L, Japan). Immediately, the mixture was centrifuged at $4000 \mathrm{rpm}$ using Hermle centrifuge (Model Ettek, Germany) for $15 \mathrm{~min}$ at room temperature $\left(25^{\circ} \mathrm{C}\right)$. The mixtures were inspected visually through cross-polarized plates for identification of physical phase appearance. The phase state was classified as isotropic (transparent), homogeneous (milky), twophase (two distinct layers observed), or multiphase regions (more than 2 layers observed) and was marked on a ternary phase diagram with one axis representing aqueous phase, the second representing oil, and the third representing a mixture of surfactant at fixed weight ratios. The phase diagrams were constructed using software, CHEMIX version 3.5 phase diagram plotter (UK).

2.4. Nanoemulsion Preparation. Table 1 shows the composition of the studied nanoemulsions. Both aqueous and oil phases were prepared separately. Phytosphingosine was dissolved in PKOEs by heating at $90^{\circ} \mathrm{C}$. Then the mixture was left to cool to room temperature before the addition of drug. Lipoid S75 which was dissolved in selected solvent (isopropanol and ethanol) at room temperature was added to the oil phase dropwise. On the other hand, the aqueous phase mixture containing Tween 20 and deionized water was heated until all Tween 20 was dissolved. At room temperature, the two phases were mixed dropwise and prehomogenised using an overhead stirrer for 4 hours using low energy emulsification method.

\subsection{Nanoemulsion Characterization}

2.5.1. Particle Size Analysis. The particle size of the nanoemulsion formulation prepared was measured by Malvern Zetasizer ZS (Malvern Instruments, Worcestershire, UK) with photon correlation spectrometer (PCS) using laser light scattering. The samples were loaded onto $1 \mathrm{~cm}^{2}$ cuvettes and were inserted into a thermostated chamber. The average of 5 measurements was taken for the particle size. All samples were measured immediately after dilution with deionized water. The graph of particle size distribution by number versus particle size was chosen and analyzed.

2.5.2. Surface Charge. The surface charge present was measured using Malvern Zetasizer ZS (Malvern Instruments, Worcestershire, UK) by laser Doppler electrophoresis. Samples were diluted with deionized water before measurement. The samples were then injected into a capillary cell for charge measurement. Zeta potential values provide information on the repulsive forces between particles in the emulsion system.

2.5.3. Stability Assessment. Prepared formulations were tested for short term stability by centrifuging at $4000 \mathrm{rpm}$ for $30 \mathrm{~min}$ [12] and were observed for phase separation. All 
TABLE 1: Compositions of nanoemulsions used in this study.

\begin{tabular}{|c|c|c|c|c|}
\hline \multirow{3}{*}{ Compound } & \multicolumn{4}{|c|}{ Content (wt.\%) } \\
\hline & \multicolumn{2}{|c|}{ Positively charged } & \multicolumn{2}{|c|}{ Negatively charged } \\
\hline & C(IPA) & $\mathrm{C}(\mathrm{EtOH})$ & C(IPA) & $\mathrm{C}(\mathrm{EtOH})$ \\
\hline \multicolumn{5}{|l|}{ Oil phase } \\
\hline Palm kernel oil ester & 10 & 10 & 10 & 10 \\
\hline (PKOEs) & 24 & 24 & 24 & 24 \\
\hline Lipoid S-75 & 24 & 24 & 24 & 24 \\
\hline Isopropanol (IPA) & 24 & - & 24 & - \\
\hline Ethanol (EtOH) & - & 24 & - & 24 \\
\hline \multicolumn{5}{|l|}{ Hydrocortisone } \\
\hline \multirow{3}{*}{ Phytosphingosine } & $0.4,0.8$ & $0.4,0.8$ & - & - \\
\hline & $1.2,1.6$ & $1.2,1.6$ & & \\
\hline & 2.0 & 2.0 & & \\
\hline \multicolumn{5}{|l|}{ Water phase } \\
\hline Tween 20 & 16 & 16 & 16 & 16 \\
\hline Preservative & 0.5 & 0.5 & 0.5 & 0.5 \\
\hline Water to & 100 & 100 & 100 & 100 \\
\hline
\end{tabular}

C(IPA): nanoemulsion with isopropanol.

$\mathrm{C}(\mathrm{EtOH})$ : nanoemulsion with isopropanol.

the formulations without significant phase separation were stored in three different temperatures: $5^{\circ} \mathrm{C}$ (refrigerator), $25^{\circ} \mathrm{C}$ (room temperature), and $45^{\circ} \mathrm{C}$ (in an incubator (Shaking Incubator DK-S1020, DAIKI Sciences Co. Ltd, Korea)). Formulations were also subjected to heat-cool cycle (6 cycles) between temperatures $4^{\circ} \mathrm{C}$ and $45^{\circ} \mathrm{C}$ with storage at each temperature not less than 24 hours. The samples were observed visually and tested for particle size and zeta potential each week for three months.

2.5.4. Viscosity Measurement. The viscosity of the nanoemulsions was measured as such without dilution using rotational viscometer RheolabQC (Anton Paar Gmbh, USA).

2.5.5. $\mathrm{pH}$ Determination. $\mathrm{pH}$ values of freshly prepared nanoemulsion formulations were measured at $25^{\circ} \mathrm{C}$ using a Delta $320 \mathrm{pH}$ meter (Mettler-Toledo, Schwerzenbach, Switzerland).

2.5.6. Conductivity. Conductivity of nanoemulsions was determined using SevenGo conductivity meter (Mettler Toledo GmbH, Switzerland).

2.5.7. Transmission Electron Microscope (TEM) Analysis. TEM was used to study the structure and morphology of the nanoemulsion. The samples were first diluted with deionized water, then dropped to 200-mesh formvar-coated copper grids, and negatively stained with uranyl acetate for $3 \mathrm{~min}$, at room temperature. Whatman filter paper was used to dry the excess liquid and prepared samples were dried at room temperature. The samples were observed with Hitachi H-7100 transmission electron microscope (Japan). The acquired digital images were processed with Adobe Photoshop software.

\section{Results and Discussions}

3.1. Selection of Composition. The most important criterion for screening of compositions is the ability of the components to solubilize the actives in the system. The components are PKOEs, water, Tween 20, Tween 40, Tween 80, isopropanol, ethanol, ethyl acetate, and phosphate buffer saline (PBS). Table 2 shows results for the solubility of hydrocortisone in PKOEs, water, phosphate buffer solution (PBS) pH 7.4, solvents, and surfactants. The solubility of hydrocortisone was found to be high in palm kernel oil esters as compared to water. As the solubility of hydrocortisone was higher in oil phase compared to aqueous phase, $\mathrm{O} / \mathrm{W}$ nanoemulsions were developed for transdermal delivery of hydrocortisone. The highest solubility of hydrocortisone was seen in Tween 20 . Therefore, Tween 20 was selected as the surfactant. As for the different solvents, the highest solubility of hydrocortisone was observed in isopropanol followed by ethanol and finally ethyl acetate. About $219.10 \pm 1.01 \mu \mathrm{g} / \mathrm{mL}$ of hydrocortisone is soluble in $1 \mathrm{~mL}$ of PBS. On the basis of the solubility studies, Tween 20, isopropanol, and ethanol, together with Lipoid S75 as an additional surfactant, were selected for the phase study.

3.2. Phase Behavior of PKOEs/Lipoid S75:Tween 20 (60:40)/Water. Components used for the construction of phase diagrams are those with better solubility of hydrocortisone. Phase behavior provides a crucial suggestion to macroscopic behavior, as it is an important factor in the thermodynamic characterization of the system. Phase behavior is a regular way to deliver the interaggregate interactions of molecule or particle on a monocular level. The primary principle is to mix the components and study the number and nature of the phases [13]. Figure 1 shows 


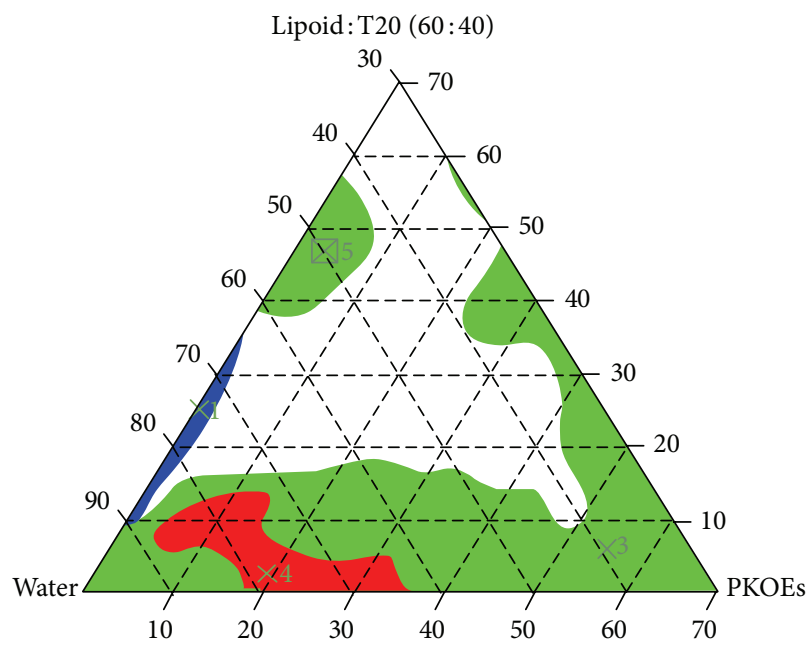

FIgURE 1: Phase behavior of PKOEs/Lipoid S75: Tween 20 (60:40)/water system at $25^{\circ} \mathrm{C}$. blue area-isotropic; white area-homogenous; green area-2 phases; red area-3 phases.

TABLE 2: Solubility of hydrocortisone in various excipients.

\begin{tabular}{lc}
\hline Excipients & Solubility $(\mu \mathrm{g} / \mathrm{mL})$ \\
\hline PKOEs & $191.61 \pm 0.99$ \\
Water & $125.20 \pm 1.23$ \\
Tween 20 & $2.35 \pm 1.05$ \\
Tween 40 & $2.01 \pm 1.12$ \\
Tween 80 & $1.87 \pm 1.09$ \\
Isopropanol & $315.25 \pm 1.00$ \\
Ethanol & $285.72 \pm 1.11$ \\
Ethyl acetate & $84.93 \pm 0.54$ \\
PBS & $219.10 \pm 1.01$ \\
\hline
\end{tabular}

Values represent mean \pm SD of three independent experiments.

the ternary phase diagram of PKOEs/Lipoid S75: Tween 20 $(60: 40) /$ water systems. Mixed binary surfactants display good mixing and hence prolong the nanoemulsion shelf life. From the phase diagram, two distinct one-phase regions consisting of solution of isotropic, transparent fluid (blue), and a homogeneous phase region (white) were detected. The remaining parts of the ternary phase diagram consist of two-phase region (green) and three-phase region (red). The ternary phase diagram was not constructed at $\geq 70 \%(\mathrm{w} / \mathrm{w})$ of oil concentration as the study was focused on the preparation of $\mathrm{O} / \mathrm{W}$ nanoemulsion in order to deliver lipophilic drug.

From Figure 1, it was observed that a small isotropic region was formed along the apex line of Lipoid S75: Tween $20(60: 40)$ and water. The composition of isotropic region contained high amount of water which led to the formation of $\mathrm{O} / \mathrm{W}$ nanoemulsions. Isotropic regions are usually referred to as a clear or transparent liquid which is expected to be stable within a long period of time. The homogeneous region was also found at the water-rich corner of the phase diagram, whereas the two-phase and three-phase region appeared mostly at the base of the ternary phase diagram at low surfactant concentration. This is due to the insufficient coverage of the particle surface with the surfactant molecules to stabilize the nanoemulsion system. At low concentration of surfactant, the amphiphiles appeared as monomers and the critical micelle concentration (CMC) is not achieved [14]. Therefore no micelles were formed. This explains the observation of two-phase and three-phase regions. Two-phase region is the largest region observed in the system.

\subsubsection{Selection of Compositions from the Ternary Phase Dia-} gram. Figure 2 illustrates the composition points selected from the ternary phase diagram. A total of nine formulations with $\leq 40 \%$ mixed surfactant $(\mathrm{w} / \mathrm{w})$ were chosen so that the drug could be incorporated into the oil phase and the compositions were listed in Table 3. Exactly 1\% (w/w) of hydrocortisone, which was kept constant in all the selected formulations, was dissolved in the oil phase. The $1 \%(\mathrm{w} / \mathrm{w})$ of hydrocortisone was made as a benchmark to compare with many commercial hydrocortisone creams. These formulations were chosen from the homogeneous region considering its suitability in pharmaceutical products. Large amount of surfactant was refrained because surfactant would lower the interfacial tension of lipids in the formulation, as well as the lipids in the skin [15]. It has been reported that an emulsion rich in surfactants is believed to cause irritancy to the skin [1].

The composition of oil phase for all nine formulations selected comprises $10 \%$ to $20 \%$ (w/w) PKOEs (Table 5). The use of relatively low amount of oil phase (palm kernel oil esters in this case) helps reduce greasy or oily feel when applied to the skin. Nanoemulsions containing PKOEs as the oil phase could accelerate skin hydration, therefore reducing skin dryness due to direct exposure to sun. On the other hand, the continuous phase typically consists of $50 \%$ to $70 \%(\mathrm{w} / \mathrm{w})$ water. The continuous phase may contain other components including thickener, water soluble surfactants, preservatives, and rheology modifier to bring benefits to the skin.

The selected nine compositions were formulated and subjected to accelerated stability test. The formulations were centrifuged at room temperature $\left(25^{\circ} \mathrm{C}\right)$ for $30 \mathrm{~min}$ at 
TABLE 3: Composition and short term stability of formulations from the homogeneous phase.

\begin{tabular}{|c|c|c|c|c|c|c|c|}
\hline Formulation & Water & PKOEs & Lipoid & Tween 20 & $\mathrm{HC}$ & Phenonip & Short term stability \\
\hline NEA & $48.5 \%$ & $20 \%$ & $18 \%$ & $12 \%$ & $1 \%$ & $0.5 \%$ & $x$ \\
\hline NEB & $48.5 \%$ & $15 \%$ & $21 \%$ & $14 \%$ & $1 \%$ & $0.5 \%$ & $x$ \\
\hline NEC & $48.5 \%$ & $10 \%$ & $24 \%$ & $16 \%$ & $1 \%$ & $0.5 \%$ & $\checkmark$ \\
\hline NED & $58.5 \%$ & $16 \%$ & $14.4 \%$ & $9.6 \%$ & $1 \%$ & $0.5 \%$ & $x$ \\
\hline NEE & $58.5 \%$ & $12 \%$ & $16.8 \%$ & $11.2 \%$ & $1 \%$ & $0.5 \%$ & $x$ \\
\hline NEF & $58.5 \%$ & $8 \%$ & $19.2 \%$ & $12.8 \%$ & $1 \%$ & $0.5 \%$ & $x$ \\
\hline NEG & $68.5 \%$ & $12 \%$ & $10.9 \%$ & $7.2 \%$ & $1 \%$ & $0.5 \%$ & $x$ \\
\hline $\mathrm{NEH}$ & $68.5 \%$ & $8 \%$ & $13.2 \%$ & $8.8 \%$ & $1 \%$ & $0.5 \%$ & $x$ \\
\hline NEI & $68.5 \%$ & $6 \%$ & $14.4 \%$ & $9.6 \%$ & $1 \%$ & $0.5 \%$ & $x$ \\
\hline
\end{tabular}

HC: hydrocortisone.

PKOES: palm kernel oil esters.

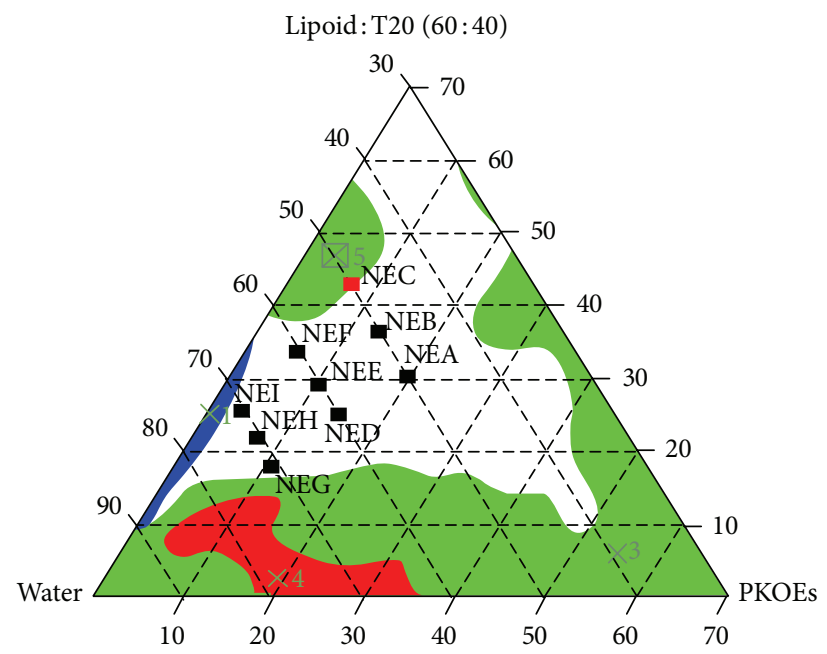

FIGURE 2: The composition points selected from the PKOEs/Lipoid: Tween 20 (60:40)/water system.

$4000 \mathrm{rpm}$. Visual observation was taken after centrifugation test for significant phase separation. Formulations with multiple phases did not proceed for further characterization. Table 3 shows the results of short term stability studies for the selected formulations. Formulation NEC was chosen for further studies as other prepared formulations showed immediate and distinct phase separation.

\subsection{Particle Size Analysis for Positively and Negatively Charged} Nanoemulsion. The mean particle size of freshly prepared (day 0 ) positively charged nanoemulsions C(IPA) $1.6 \%$ PS and $\mathrm{C}(\mathrm{EtOH}) 1.6 \%$ PS were $200.7 \mathrm{~nm}$ and $210.5 \mathrm{~nm}$, respectively. However, further increase in percentage of phytosphingosine to $2.0 \%$ showed no changes in droplet size. This is because $1.6 \%$ of phytosphingosine is sufficient to fully occupy the particle surface. At the day of production the mean particle size for negatively charged nanoemulsions was $185.9 \mathrm{~nm}$ for $\mathrm{C}(\mathrm{IPA})$ and $198.00 \mathrm{~nm}$ for $\mathrm{C}(\mathrm{EtOH})$.

Due to the fact that the only difference between the positively and negatively charged nanoemulsions is the presence of phytosphingosine, the increase in particle size is most likely being affected by the substance itself. The nature of phytosphingosine being a poorly soluble substance explains the increase in particle size for the positively charged nanoemulsion compared to the negatively charged nanoemulsion.

3.4. Effect of Phytosphingosine on Zeta Potential. Generally, zeta potential values, which exceed $30 \mathrm{mV}$ regardless of the positive or negative prefix, are considered as stable nanoemulsions. Table 4 illustrates the zeta potential values of all negatively and positively charged nanoemulsion. From the results, we can deduce that increasing phytosphingosine concentration from 0.4 to $2.0 \%$ led to a change in zeta potential value from negative value to positive. This proved that phytosphingosine contributes to the positive charge of the nanoemulsion.

Further increase in phytosphingosine concentration however results in sedimentation of phytosphingosine. One of the disadvantages of phytosphingosine is that it has very low solubility [3]. Therefore, in our studies, heat is applied to increase the solubility of phytosphingosine before homogenization. Nevertheless this failed to occur for formulation $\mathrm{C}(\mathrm{EtOH}) 2.0 \%$ PS. Precipitation of phytosphingosine was observed even after application of heat after a week of storage.

As for the negatively charged nanoemulsions (C(IPA) and $\mathrm{C}(\mathrm{EtOH})$ ) both showed zeta potential values over $-30 \mathrm{mV}$. 
TABLE 4: Zeta potential and $\mathrm{pH}$ values of negatively and positively charged nanoemulsions.

\begin{tabular}{lcc}
\hline Formulations & Zeta potential $(\mathrm{mV})$ & $\mathrm{pH}$ \\
\hline C(IPA $)$ & -53.2 & 4.48 \\
C(EtOH) & -58.6 & 4.54 \\
C(IPA) $0.4 \% P S$ & -48.2 & 5.51 \\
C(IPA)0.8\%PS & -19.7 & 5.48 \\
C(IPA)1.2\%PS & +11.0 & 6.21 \\
C(IPA)1.6\%PS & +25.9 & 5.49 \\
C(IPA)2.0\%PS & +26.0 & 6.38 \\
C(EtOH)1.6\%PS & +24.0 & 5.50 \\
C(EtOH)2.0\%PS & +24.3 & 5.54 \\
\hline
\end{tabular}

TABLE 5: Conductivity measurements of nanoemulsions.

\begin{tabular}{lc}
\hline Formulations & Conductivity $(\mu \mathrm{S} / \mathrm{cm})$ \\
\hline C(IPA) & 56.2 \\
C(EtOH) & 65.3 \\
C(IPA) $0.4 \% P S$ & 61.5 \\
C(IPA) $0.8 \% P S$ & 55.6 \\
C(IPA)1.2\%PS & 75.9 \\
C(IPA)1.6\%PS & 76.4 \\
C(IPA) $2.0 \% P S$ & 79.7 \\
C(EtOH)1.6\%PS & 59.8 \\
C(EtOH) $2.0 \% P S$ & 60.1 \\
\hline
\end{tabular}

Consequently, taken into consideration the surface charge and particle size of all formulations, C(IPA), C(EtOH), C(IPA) $1.6 \%$ PS, and $\mathrm{C}(\mathrm{EtOH}) 1.6 \%$ PS were chosen for further stability studies.

3.5. $p H$ Studies. $\mathrm{pH}$ values of the freshly prepared formulations are shown in Table 2. Since skin $\mathrm{pH}$ is in the range of 5 to 6 , hence topical formulations should also have a $\mathrm{pH}$ value approximately in that range [16]. This corresponds to the $\mathrm{pH}$ of our nanoemulsions. In addition, at $\mathrm{pH}$ below 9 the amino group in phytosphingosine gets protonated. Thus, it explains the positive charge of the nanoemulsion [5].

3.6. Conductivity. Conductivity of the nanoemulsions was measured to determine the phase system (o/w or w/o) of the nanoemulsion [17]. Oil-in-water nanoemulsions are highly conducting because water is in the external phase. On the other hand, water-in-oil nanoemulsion, where water is in the internal phase is less conducting [18]. From the conductivity results in Table 5 the nanoemulsion formulations were detected as oil-in-water nanoemulsions.

3.7. Viscosity Determination. The viscosity of all prepared formulations was determined (Table 6). All formulations showed almost similar viscosity measurement. This is because the compositions of all the formulations do not vary much. Generally, the viscosity of all formulations was very low. However, this coincides with one of the characteristics of nanoemulsion which is low viscosity [19].
TABLE 6: Viscosity of nanoemulsion formulations.

\begin{tabular}{lc}
\hline Formulations & Viscosity (mPas) \\
\hline C(IPA) & 54.0 \\
C(EtOH) & 62.4 \\
C(IPA) $0.4 \% P S$ & 77.4 \\
C(IPA) $0.8 \% P S$ & 106.0 \\
C(IPA)1.2\%PS & 59.2 \\
C(IPA)1.6\%PS & 67.2 \\
C(IPA)2.0\%PS & 64.9 \\
C(EtOH)1.6\%PS & 59.7 \\
C(EtOH)2.0\%PS & 62.5 \\
\hline
\end{tabular}

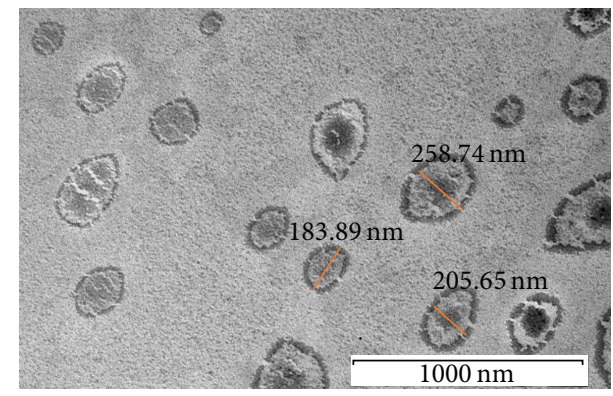

FIGURE 3: TEM image of C(IPA) $1.6 \%$ PS formulation.

3.8. Particle Morphology. TEM analysis was carried out to confirm that the nanoemulsion droplets of all formulations were spherical and particle sizes measured are in accordance with Malvern Zetasizer ZS. All nanoemulsions showed similar TEM micrograph. Figure 3 shows the TEM image of a positively charged nanoemulsion C(IPA) $1.6 \%$ PS as a representative of all. As shown in Figure 3, the droplets of C(IPA) $1.6 \%$ PS were spherical in shape. In addition, the particle size measured in TEM is consistent with the size obtained using photon correlation spectroscopy.

3.9. Long Term Stability. Figure 4 shows the particle size for formulations C(IPA), C(EtOH), C(IPA) $1.6 \%$ PS, and $\mathrm{C}(\mathrm{EtOH}) 1.6 \%$ PS over a period of $3 \mathrm{~m}$. No formulations showed significant difference in particle size over time. This proved that the negatively charged nanoemulsion has the ability to overcome all instability phenomena. This could be due to the presence of solvent that promotes the solubility of oil in aqueous phase. Low solubility of oil in aqueous phase could result in Ostwald ripening process, the main cause of instability in nanoemulsion [5]. This stability also means there is sufficient surfactant surrounding the particle surface.

On the other hand, the presence of phytosphingosine most likely explains that the particle size of the positively charged nanoemulsions remained consistently under $300 \mathrm{~nm}$. With the additional cosurfactant, there is no doubt that the particles surface achieved maximal occupation. Besides that, phytosphingosine which has an amphiphilic structure could arrange itself to form a mixed film at the oil and water interface, thus resolving the nanoemulsion instability issue. 


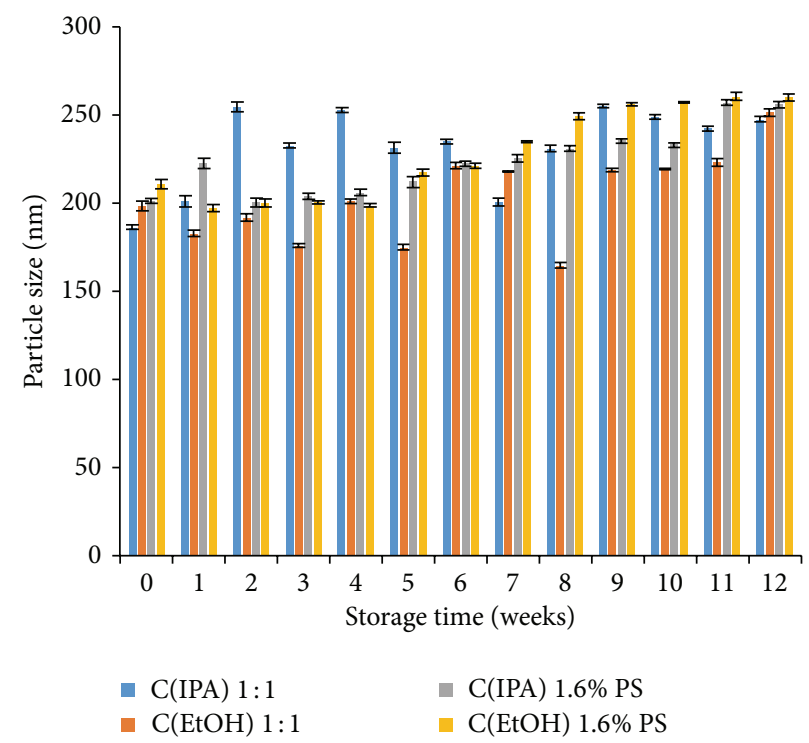

FIGURE 4: The mean particle size of hydrocortisone loaded NEC with different solvent over a period of $3 \mathrm{~m}$. ( 0 week refers to freshly prepared nanoemulsion). C(IPA): 24.5\% water, 10\% palm kernel oil esters (PKOEs), 24\% Lipoid S75, 16\% Tween 20, 24\% IPA 1.0\% hydrocortisone, and 0.5\% phenonip; C(EtOH): 24.5\% water, 10\% palm kernel oil esters (PKOEs), 24\% Lipoid S75, 16\% Tween 20, 24\% EtOH, 1.0\% hydrocortisone, and $0.5 \%$ phenonip; C(IPA) $1.6 \%$ PS: $22.9 \%$ water, $10 \%$ palm kernel oil esters (PKOEs), 24\% Lipoid S75, 16\% Tween 20, 24\% IPA, 1.6\% phytosphingosine, $1.0 \%$ hydrocortisone; and $0.5 \%$ phenonip C(EtOH) 1.6\% PS: $22.9 \%$ water, $10 \%$ palm kernel oil esters (PKOEs), $24 \%$ Lipoid S75, $16 \%$ Tween $20,24 \%$ IPA, $1.6 \%$ phytosphingosine, $1.0 \%$ hydrocortisone, and $0.5 \%$ phenonip.

\section{Conclusion}

Stable negatively and positively charged nanoemulsions were produced using low energy emulsification method. Phytosphingosine was added as a cosurfactant in order to induce the positive charge in the nanoemulsion. A range of percentage $(0.4 \%-2.0 \%)$ of phytosphingosine was added to study the physicochemical properties of the nanoemulsion formulations. From the results it is shown that $1.6 \%$ phytosphingosine obtained the best zeta potential and particle size value.

The $\mathrm{pH}$ obtained not only was compatible with the skin $\mathrm{pH}$ but also shows a $\mathrm{pH}$ lower than 9 which is when the amino group in phytosphingosine gets protonated hence producing a positive charge. The conductivity results proved that the formulations are oil-in-water system. In addition, TEM micrographs showed similar particle size with the size obtained using Zetasizer.

It can be concluded that positively and negatively charged nanoemulsions have great potential in transdermal delivery system. Since from this work promising results were obtained, further investigation of the drug release and drug penetration of both positively and negatively charged nanoemulsions will be studied to further prove the efficacy of nanoemulsion with hydrocortisone as a delivery system for dermal application.

\section{Conflict of Interests}

The authors declare that there is no conflict of interests regarding the publication of this paper.

\section{Acknowledgments}

The authors would like to acknowledge financial support from Graduate Research fellowship (GRF), Universiti Putra Malaysia, and the Ministry of science, Technology and Innovation (MOSTI) science fund for funding this research.

\section{References}

[1] F. Shakeel, S. Baboota, A. Ahuja, J. Ali, M. Aqil, and S. Shafiq, "Nanoemulsions as vehicles for transdermal delivery of aceclofenac," AAPS PharmSciTech, vol. 8, no. 4, article E104, 2007.

[2] F. Shakeel and W. Ramadan, "Transdermal delivery of anticancer drug caffeine from water-in-oil nanoemulsions," Journal of Colloids and Surfaces B: Biointerfaces, vol. 75, no. 1, pp. 356$362,2010$.

[3] Y. Baspinar and H. Borchert, "Penetration and release studies of positively and negatively charged nanoemulsions-is there a benefit of the positive charge?" International Journal of Pharmaceutics, vol. 430, no. 1-2, pp. 247-252, 2012.

[4] H. Trommer and R. H. H. Neubert, "Overcoming the stratum corneum: the modulation of skin penetration," Skin Pharmacology and Physiology, vol. 19, no. 2, pp. 106-121, 2006.

[5] E. Yilmaz and H. Borchert, "Design of a phytosphingosinecontaining, positively-charged nanoemulsion as a colloidal carrier system for dermal application of ceramides," European Journal of Pharmaceutics and Biopharmaceutics, vol. 60, no. 1, pp. 91-98, 2005.

[6] J. Arikawa, M. Ishibashi, M. Kawashima, Y. Takagi, Y. Ichikawa, and G. Imokawa, "Decreased levels of sphingosine, a natural 
antimicrobial agent, may be associated with vulnerability of the stratum corneum from patients with atopic dermatitis to colonization by Staphylococcus aureus," Journal of Investigative Dermatology, vol. 119, no. 2, pp. 433-439, 2002.

[7] B. Melnik, "Disturbances of antimicrobial lipids in atopic dermatitis," Journal der Deutschen Dermatologischen Gesellschaft, vol. 4, no. 2, pp. 114-123, 2006.

[8] P. W. Wertz, D. C. Swarzendruber, K. C. Madison, and D. T. Downing, "Composition and morphology of epidermal cyst lipids," Journal of Investigative Dermatology, vol. 89, no. 4, pp. 419-425, 1987.

[9] K. Bouchemal, S. Briançon, E. Perrier, and H. Fessi, "Nanoemulsion formulation using spontaneous emulsification: solvent, oil and surfactant optimisation," International Journal of Pharmaceutics, vol. 280, no. 1-2, pp. 241-251, 2004.

[10] S. Baboota, F. Shakeel, A. Ahuja, J. Ali, and S. Shafiq, "Design, development and evaluation of novel nanoemulsion formulations for transdermal potential of celecoxib," Acta Pharmaceutica, vol. 57, no. 3, pp. 315-332, 2007.

[11] A. López, F. Llinares, C. Cortell, and M. Herráez, "Comparative enhancer effects of Span 20 with Tween 20 and Azone on the in vitro percutaneous penetration of compounds with different lipophilicities," International Journal of Pharmaceutics, vol. 202, no. 1-2, pp. 133-140, 2000.

[12] D. Mou, H. Chen, D. Du et al., "Hydrogel-thickened nanoemulsion system for topical delivery of lipophilic drugs," International Journal of Pharmaceutics, vol. 353, no. 1-2, pp. 270-276, 2008.

[13] V. Pillai and D. O. Shah, Dynamic Properties of Interfaces and Association Structures, AOCS Press, Urbana, Ill, USA, 1996.

[14] L. D. T. Santos, E. L. B. Neto, M. C. P. A. Moura, T. N. C. Dantas, and A. A. D. Neto, "Molecular behavior of ionic and nonionic surfactants in saline medium," Journal of Colloids and Surfaces A: Physicochemical Engineering Aspects, vol. 333, pp. 156-162, 2009.

[15] L. Mitchell and B. A. Schlossman, The Chemistry and Manufacture of Cosmetic, Allured, 2000.

[16] N. Akhtar, M. Ahmad, H. M. S. Khan et al., "Formulation and characterization of multiple emulsion containing $1 \% \mathrm{~L}$-ascorbic acid," Bulletin of the Chemical Society of Ethiopia, vol. 24, no. 1, pp. 1-10, 2010.

[17] N. U. Okur, S. Apaydin, N. Ü. K. Yavaşoğlu, A. Yavaşoğlu, and H. Y. Karasulu, "Evaluation of skin permeation and anti-inflammatory and analgesic effects of new naproxen microemulsion formulations," International Journal of Pharmaceutics, vol. 416, no. 1, pp. 136-144, 2011.

[18] V. Devarajan and V. Ravichandran, "Nanoemulsions: as modified drug delivery tool," International Journal of Comprehensive Pharmacy, vol. 2, no. 4, pp. 1-6, 2011.

[19] S. Baboota, A. Alazaki, K. Kohli, J. Ali, N. Dixit, and F. Shakeel, "Development and evaluation of a microemulsion formulation for transdermal delivery of terbinafine," PDA Journal of Pharmaceutical Science and Technology, vol. 61, no. 4, pp. 276-285, 2007. 

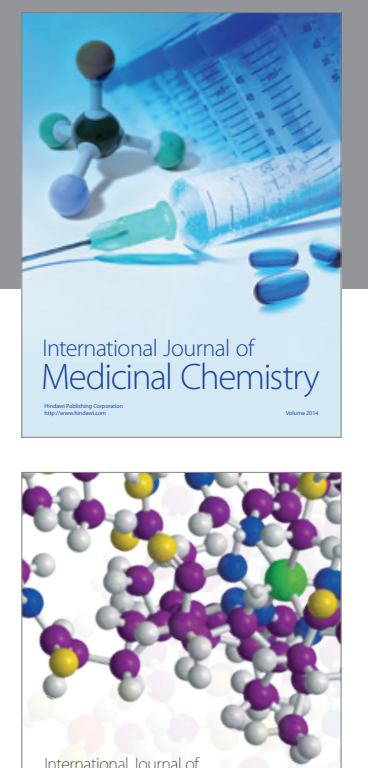

\section{Carbohydrate} Chemistry

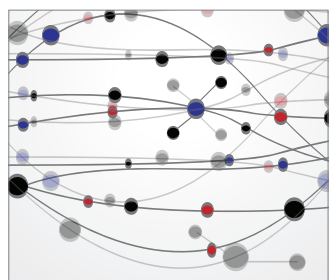

The Scientific World Journal
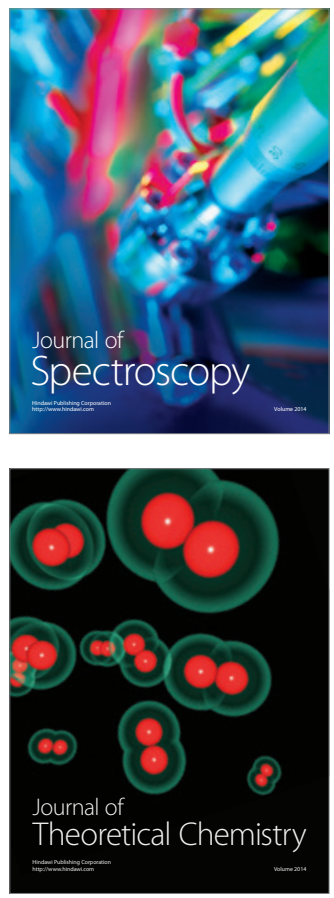
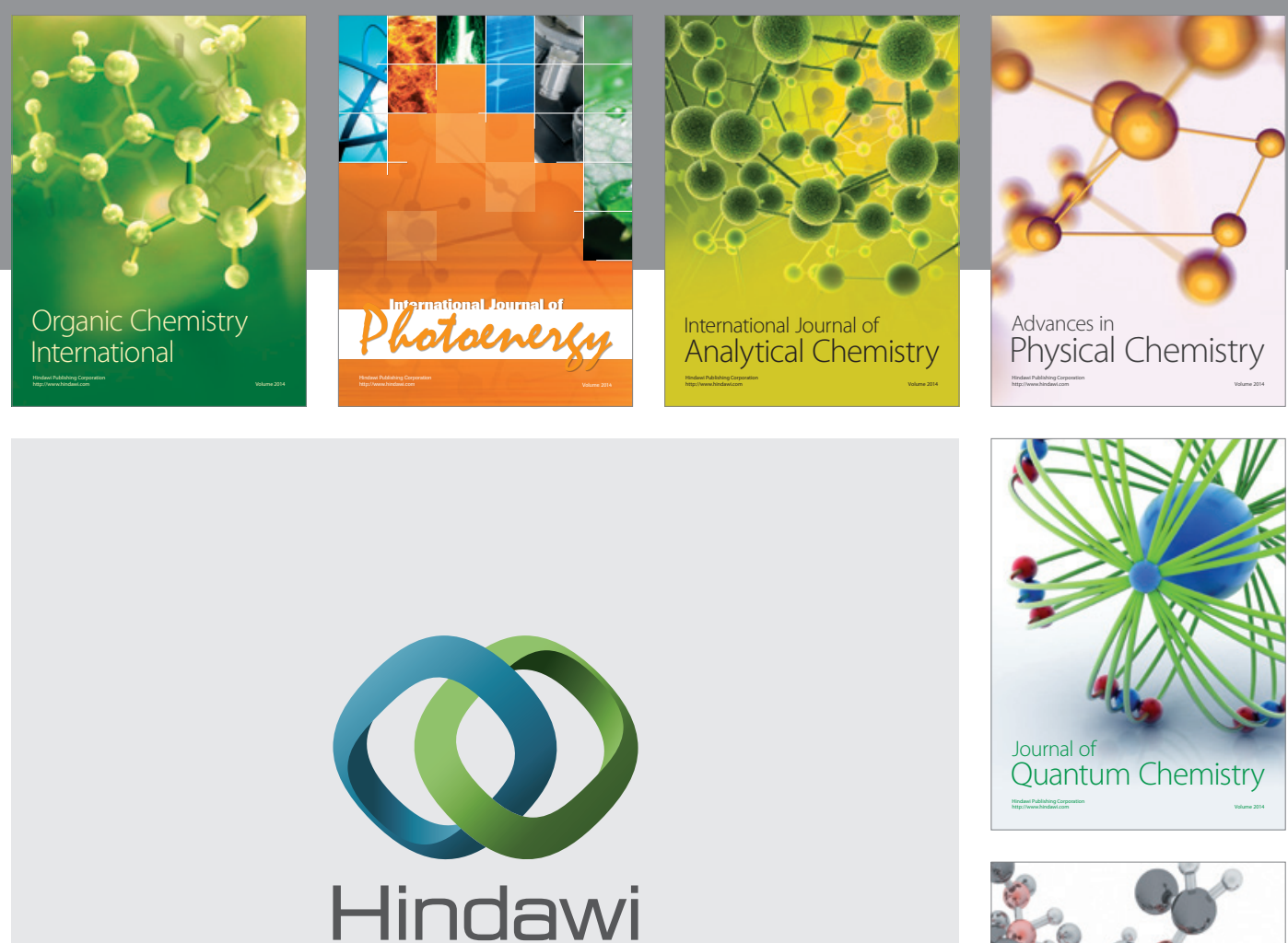

Submit your manuscripts at

http://www.hindawi.com

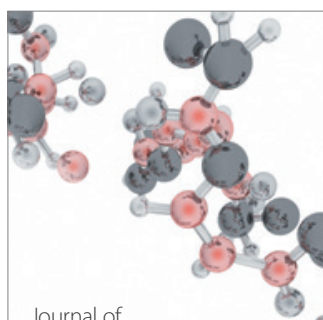

Analytical Methods

in Chemistry

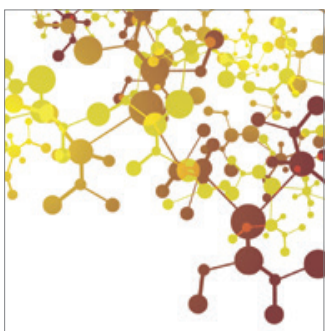

Journal of

Applied Chemistry

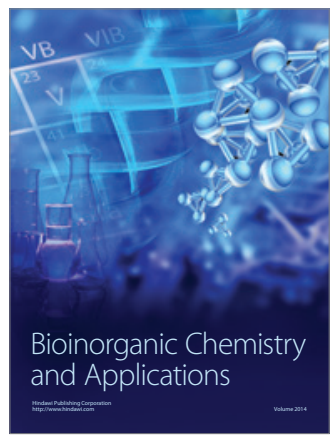

Inorganic Chemistry
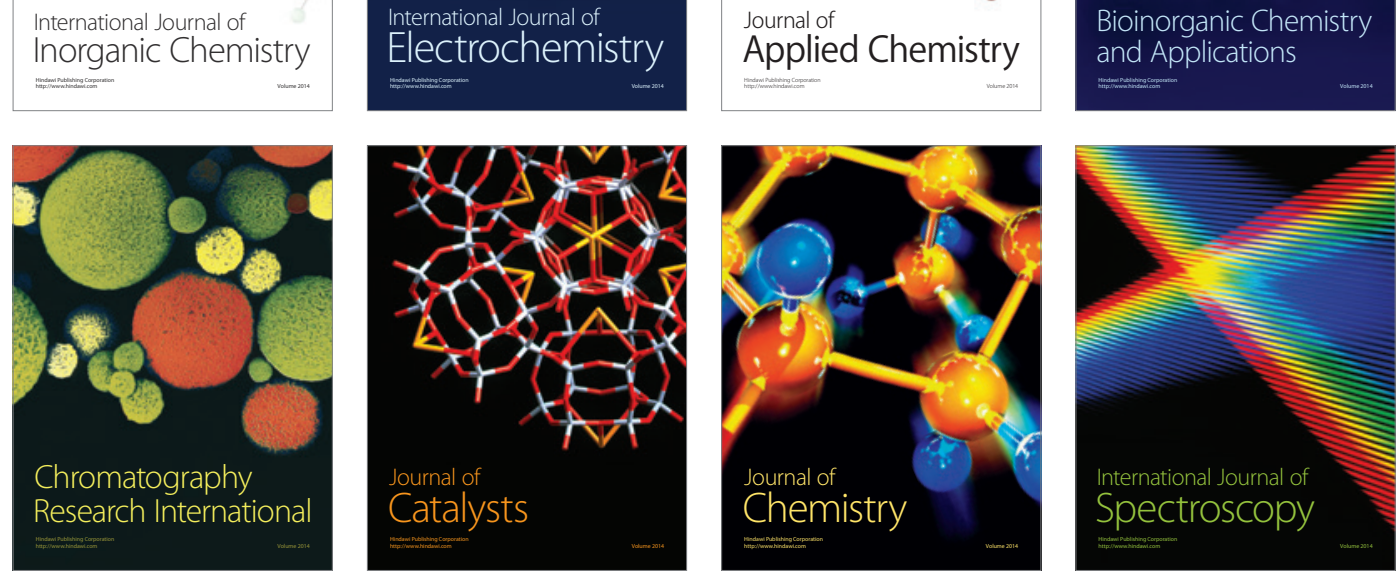